\title{
Polyethylene glycol derived carbon quantum dots nanofluids: An excellent lubricant for diamond-like carbon film/bearing steel contact
}

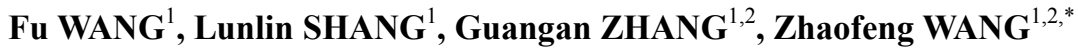 \\ ${ }^{1}$ State Key Laboratory of Solid Lubrication, Lanzhou Institute of Chemical Physics, Chinese Academy of Sciences, Lanzhou 730000 , China \\ ${ }^{2}$ Center of Materials Science and Optoelectronics Engineering, University of Chinese Academy of Sciences, Beijing 100049, China \\ Received: 22 May 2021 / Revised: 30 July 2021 / Accepted: 15 August 2021
}

(C) The author(s) 2021.

\begin{abstract}
Polyethylene glycol derived carbon quantum dots nanofluids were synthesized via a slow thermal oxidation process. The size of carbon quantum dots was ca. $2 \mathrm{~nm}$ and had a decreasing trend with the increase of oxidation time. When used as lubricant in a diamond-like carbon film/bearing steel interface, the nanofluids achieved an ultra-low friction coefficient $(\mu \approx 0.02)$, much lower than that of original polyethylene glycol $(\mu=0.12)$. The worn surface analyses revealed that the nanofluids could effectively inhibit the tribo-oxidation of steel counterpart that occurred under original polyethylene glycol lubrication, and hence reduced the abrasion component of friction. Especially, the poly-hydroxyl carbon dots and oxidized polyethylene glycol species in nanofluids induced a hydroxyl-rich sliding interface via their tribochemical reactions with friction surfaces, which promoted the adsorption of polyethylene glycol molecules on sliding surfaces. Along with the mild corrosion wear of steel counterface, this shifted the boundary lubrication to a mixed/film lubrication regime, thereby achieving an ultra-low friction coefficient. The above results suggest that the polyethylene glycol derived carbon quantum dots nanofluids should be a quite excellent candidate lubricant for solid-liquid synergy lubrication based on diamond-like carbon films.
\end{abstract}

Keywords: polyethylene glycol; diamond-like carbon films; ultra-low friction; synergy lubrication

\section{Introduction}

Fuel economy is becoming increasingly important due to the global energy issues. To address this issue, tribological scientists are actively exploring advanced lubricating materials and technologies to supply a low-friction contact in mechanical interfaces [1-3]. As a promising route, solid-liquid synergy lubrication causes strong interest in scientific and industrial communities [4]. It not only provides great potential in improving the durability of components but also opens an opportunity for low-friction sliding systems. Inspired by such tribological potential, solid-liquid synergy lubrication systems have been investigated in recent years toward the specific applications [5-8].

Diamond-like carbon (DLC) films represent a class of outstanding solid lubricating materials, which possess high hardness, low friction coefficient, and excellent anti-wear property. They find wide applications in many aeras, such as car parts, magnetic storage disks, micro/nano-electromechanical devices, and biomedicine [9]. Considering their outstanding tribological properties, lots of researches have paid attention to solid-liquid synergy lubrication based on DLC films [5]. Different DLC films (e.g., tetrahedral amorphous carbon, graphite-like films, and elementdoped DLC films) are investigated under varying lubrications (e.g., water, glycerol, oleic acid, base oil,

* Corresponding author: Zhaofeng WANG, E-mail: zhfwang@licp.cas.cn 
ion liquid, and fully formulated engine oil) [10-17]. It is demonstrated that the tribological properties of DLC-based solid-liquid synergy systems are strongly affected by lubricating media, contact conditions, and the characteristics of DLC films (such as hydrogen content, $\mathrm{sp}^{2} / \mathrm{sp}^{3}$ carbon ratio, and doping element). DLC films can achieve outstanding lubricity under glycerol and oleic acid lubrication [16, 18]. Especially, DLC films with high $\mathrm{sp}^{3}$-hybrid carbon exhibit ultra-low friction coefficients $(<0.03)$ under glycerol, oleic acid, and even poly- $\alpha$-olefin lubrications $[12,18$, 19]. A variety of additives including traditional and nano ones are examined in DLC-based solid-liquid synergy lubrication. The deteriorated or improved tribological performances are observed relying on the specific conditions. It is found that some friction modifiers (e.g., oleic acid, alkyl oleate, and glycerol mono-oleate) show excellent friction-reducing ability in lubricated DLC contacts [10, 16, 20]. A -OH terminated carbon surface is usually related to the ultra-low friction behavior [21]. Nevertheless, it should be noted that most traditional lubricants used for steel/steel contacts are not ideal for DLC-based solid-liquid synergy lubrication because of the chemical difference between carbon and steel. Thus, it is very necessary to develop suitable lubricants toward DLC-based solid-liquid lubrication.

Polyethylene glycol (PEG), an eco-friendly and degradable polyether lubricant, has found a wide range of industrial applications, such as textile lubricant, compressor oil, metal working liquids, brake liquids, and gear oils [22, 23]. Compared to hydrocarbon lubricants, its $-\mathrm{OH}$ groups are expected to offer potential tribochemical modification to inert DLC surfaces during friction. This makes it possible as a candidate lubricant for DLC-based solid-liquid lubrication. However, PEG itself usually shows relatively poor lubricity under boundary conditions, offering a moderate friction coefficient (ca. $\mu=0.2$ ) [24]. Many traditional lipophilic additives, such as carboxylic acid, ester, borate, and organomolybdenum compounds, are incompatible with PEG base liquid. As a result, various new additives were investigated in PEG fluid recently, which can significantly improve the lubricating performances of PEG in steel/steel contacts $[22,24-26]$. Among them, carbon quantum dots (CQDs) as an emerging additive in PEG base liquid can provide excellent friction-reducing and anti-wear abilities in steel/steel contacts [27, 28]. This arouses the interest in advanced PEG-based lubricants, especially considering the flexibility in chemical composition and structure of CQDs. In other words, there may be endless possibilities to produce excellent PEG lubricants when versatile CQDs are used as additives. However, up to now, there are few studies to investigate the PEG lubricant in a DLC contact.

Herein, in consideration of the great potential of DLC films in solid-liquid synergy lubrication, we aim to develop an excellent lubricant used for DLC film/bearing steel interface, an important industrial interface. Combining the outstanding anti-friction and anti-wear potentials of CQDs in PEG base liquid and the recent advance in the synthesis of PEG-derived carbon dots $[29,30]$, in this work, we optimized the lubricity of PEG base fluid through in-situ generating PEG-derived CQDs, offering a more efficient and economic pathway to obtain excellent PEG lubricants for DLC film/bearing steel contact. It was shown that, without any additional reagents, a slow thermal oxidation treatment could directly produce CQDs in PEG base fluid. Such PEG-derived CQDs nanofluids provided outstanding lubricity at DLC/bearing steel interface.

\section{Experimental procedure}

\subsection{Materials}

Polyethylene glycol 200 (PEG200) was purchased from Chron Chemicals Co., Ltd. (Chengdu, China). Hydrogen-free diamond-like carbon (DLC) films in this work were deposited on highly polished 316L stainless steel ( $R_{\mathrm{a}}<30 \mathrm{~nm}$, for common friction tests) and GCr15 bearing steel substrates $\left(R_{\mathrm{a}}<30 \mathrm{~nm}\right.$, for friction-load experiments) using a closed field unbalanced magnetron sputtering system (UDP650, Teer Coatings Ltd.) with graphite targets and chromium targets. The detailed deposition procedure was seen in the previous work by $\mathrm{Xu}$ et al. [31]. The DLC film has a thickness of ca. $2 \mu \mathrm{m}$, a hardness of $14.8 \mathrm{GPa}$, and an elastic modulus of $158 \mathrm{GPa}$. It shows a high $I_{\mathrm{D}} / I_{\mathrm{G}}$ value in Raman spectrum (Fig. 1), suggesting 


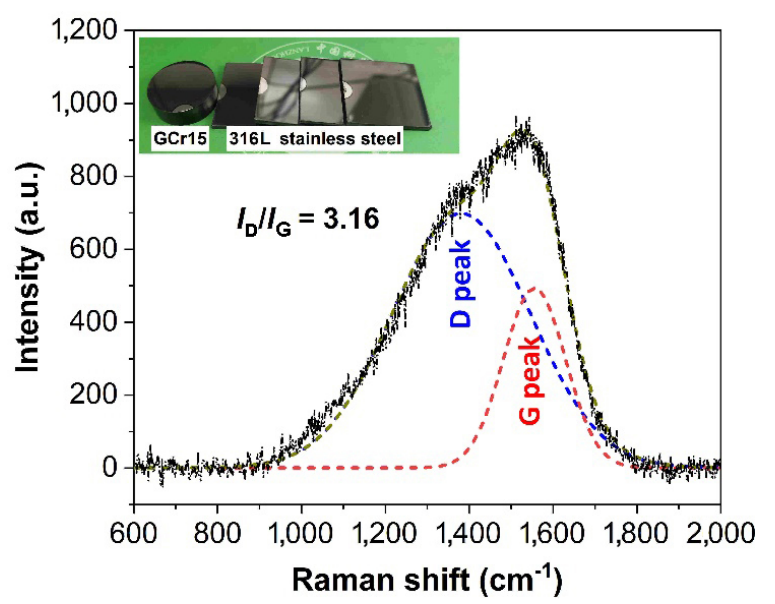

Fig. 1 Raman spectrum of the as-prepared DLC film. The inset is the photographs of DLC film on steel substrates.

that the as-deposited DLC film contains high $\mathrm{sp}^{2}$ hybrid carbon, also called graphite-like carbon (GLC) film.

\subsection{Synthesis of PEG derived carbon quantum dots nanofluids}

PEG carbon quantum dots (PEG-CQDs) nanofluids in this work were synthesized via a slow thermal oxidation process. Specifically, PEG200 in a $100 \mathrm{~mL}$ round-bottom flask was heated to $120^{\circ} \mathrm{C}$ in laboratory air under slow agitation for 10, 20, 30, 40, 50, 60, 70, and $110 \mathrm{~h}$, respectively. After cooling to room temperature, the treated PEGs (nanofluids) were used as lubricants without any further treatment. The PEG-CQDs nanofluids were denoted as PEG- $X \mathrm{~h}$, where $X$ referred to the treatment time.

\subsection{Testing methods}

Tribological evaluation was performed on a ball-on-disc CSM tribometer (Anton Paar) in a reciprocating sliding mode. Friction pairs consisted of DLC-coated steel disk and $6 \mathrm{~mm}$ diameter of balls (made of GCr15 bearing steel, 316L stainless steel, Brass, or Zirconia) with smooth surfaces $\left(R_{\mathrm{a}}<50 \mathrm{~nm}\right)$. Before testing, both the balls and disks were cleaned with ethanol and hexane successively for $10 \mathrm{~min}$ in an ultrasonic bath. Tests were carried out at a frequency of $5.0 \mathrm{~Hz}$, a sliding stroke of $5.0 \mathrm{~mm}$, and a total sliding distance of $200 \mathrm{~m}$ in ambient air (relative humidity, $20 \%-30 \%$ ). The normal load was from 5 to $20 \mathrm{~N}$. Lubricant was introduced into the sliding interface with a volume of $200 \mu \mathrm{L}$. Dynamic friction coefficient was measured by a transducer linear variable differential transformer (LVDT). The average friction coefficient of each test was calculated based on all measurement data in a $200 \mathrm{~m}$ sliding. Three independent friction tests were carried out to give the standard deviation for each sample. The wear track profile formed on DLC films was measured with a 2D surface profiler (Alpha-step D-100, KLA-Tencor, USA). The specific wear rates were calculated by the following formula:

$$
W_{\mathrm{r}}=V /(L \times D)
$$

where $V$ is the volume loss of DLC films $\left(\mathrm{mm}^{3}\right), L$ is the applied loads $(\mathrm{N})$, and $D$ is the total sliding distance $(\mathrm{m})$. All tests were conducted independently at least three times.

\subsection{Characterizations}

The hardness and elastic modulus of DLC film were measured by a nanoindentation instrument (TTX-NHT2, Anton-Paar) under a load of $4 \mathrm{mN}$. Three points were tested to obtain a reliable result. Indentation depth was less than one tenth of the DLC film thickness to reduce the effect of steel substrate. Fluorescence spectra were measured using a fluorescence spectrometer (OmniFluo, Zolix, China) under excitation from 300 to $400 \mathrm{~nm}$. Fourier transform infrared (FT-IR) spectra were recorded on a Nexus 870 IR spectrometer ranging from 400 to $4,000 \mathrm{~cm}^{-1}$. Ultraviolet-visible (UV-vis) absorption spectra were obtained on a spectrometer (PE lamda 950, USA) using air as a reference. Transmission electron microscopy (TEM) analysis was carried out using a JEOL JEM 2100 system operated at $200 \mathrm{kV}$. The TEM samples were prepared through dropping the ethanol solution of PEG onto ultra-thin carbon film coated copper grids. The wear tracks formed on DLC films and the wear scars formed on steel balls were observed by an optical microscope (ZEISS, AX10-Axio). Raman spectra were acquired from worn DLC and steel ball surfaces using a 50× objective on a Renishaw InVia Raman microscope with a $532 \mathrm{~nm}$ laser. A capillary viscometer (SYP1003-III, capillary inner diameter of $1.0 \mathrm{~mm}$, viscometer constant of $0.06632 \mathrm{~mm}^{2} / \mathrm{s}^{2}$ ) was used to measure the kinetic viscosity of the original and treated PEGs at $40^{\circ} \mathrm{C}$, as in Table 1. 
Table 1 Kinetic viscosity of the original and treated PEGs at $40{ }^{\circ} \mathrm{C}$.

\begin{tabular}{cc}
\hline Samples & Kinetic viscosity $\left(\mathrm{mm}^{2} / \mathrm{s}\right)$ \\
\hline PEG200 & 23.74 \\
PEG-10h & 23.63 \\
PEG-20h & 23.71 \\
PEG-30h & 23.72 \\
PEG-40h & 23.70 \\
PEG-60h & 23.66 \\
PEG-70h & 23.72 \\
PEG-110h & 23.74 \\
\hline
\end{tabular}

\section{Results and discussion}

PEG200, a biocompatible non-conjugated polymer, has colorless and transparent liquid appearance. It has been frequently used for the synthesis of carbon dots as carbon source, passivating agent and solvent, via direct heating, hydrothermal, ultrasonication, microwave, and electrolysis methods [30, 32]. Herein, we adopted a slow thermal oxidation route to synthesize the PEG-derived carbon dots at a lower temperature, as shown in Fig. 2(a), which avoided the production of a dark-colored PEG liquid. After the slow thermal oxidation, it was seen that PEG retained its original appearance, only very pale yellow with the increase of thermal oxidation time (Fig. 2(b)). The treated PEGs also showed exactly the same kinetic viscosity with original PEG200 (Table 1). Strong blue fluorescence emission was noted for the thermally treated PEGs under ultraviolet light, as shown in Fig. 2(c), suggesting the generation of PEG-derived CQDs. It is considered that PEG molecules can undergo a "pyrolysis-clustering-carbonization" process during the formation of carbon dots. They are firstly broken down and oxidized into various fragments (i.e., polymeric radicals, carbonyls, hydroxyls, and alkenes) in the presence of oxygen. Then, the alkene fragments aggregate to form the hydrophobic cores and grow into larger clusters. Hydrophilic oxygen-containing fragments (i.e., ethers, hydroxyls, and carbonyls) tend to stay around the cluster surface to form surface passivation layers [30]. Evidently, the PEG-derived CQDs have different structure and composition from those dark-colored CQDs derived from citric acid in Refs. [22, 25, 27, 28]. Also, the nearly-colorless feature is of key importance for their application as additive,

(a)
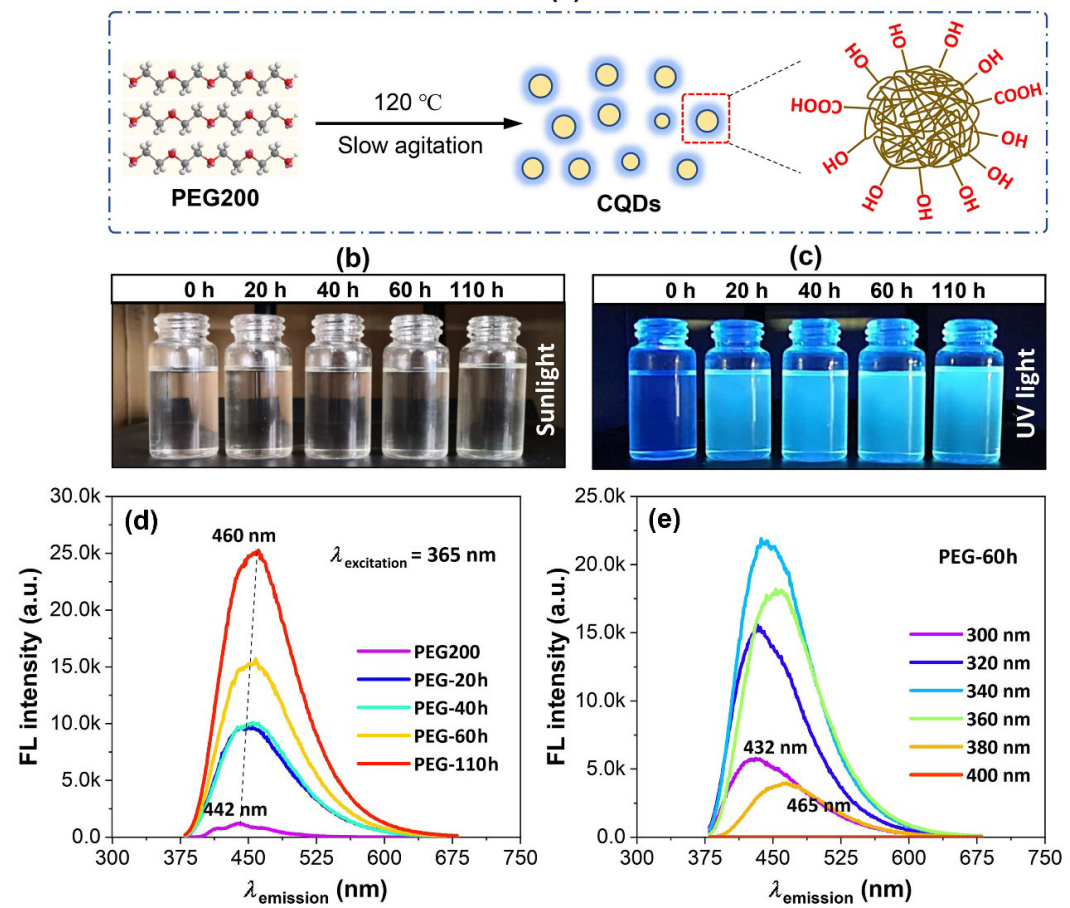

Fig. 2 (a) Schematic illustration of the thermal oxidation of PEG. Photographs of the PEG200 and treated PEGs under (b) sunlight and (c) ultraviolet light (365 nm). Fluorescence spectra of (d) PEG200 and treated PEGs under excitation at $365 \mathrm{~nm}$ and (e) PEG-60h under varying excitations. 
considering the color change as an indicator of lubricant decay.

Fluorescence spectra were measured to investigate the characteristic emission of the thermally-treated PEGs. Figure 2(d) shows the fluorescence (FL) spectra of the original PEG200 and treated PEGs. The original PEG200 also shows a faint blue fluorescence emission under $365 \mathrm{~nm}$ excitation, possibly due to the unknown impurities. After thermal oxidation, the emission intensity of the PEGs is remarkably enhanced, and the optimal emission peak shifts from 442 to $460 \mathrm{~nm}$ with the increase of oxidation time. The full width at half maximum (FWHM) of FL spectra of the treated PEGs is ca.100 nm, which is consistent with that of most reported carbon dots [29]. As seen in Fig. 2(e), with an increase in the excitation wavelength from 300 to $400 \mathrm{~nm}$, the optimal emission peak of the PEG-60h shifts to higher wavelengths, and the emission intensity firstly increased and then decreased. This is mainly due to the different size of carbon dots and a considerable distribution of emissive trap sites on each carbon dot.

TEM analysis was carried out to further characterize the thermally-treated PEGs, as shown in Fig. 3. It is clear that a large quantity of CQDs was produced during the thermal oxidation. According to the low magnification images (Figs. 3(a)-3(d)), the CQDs were prone to form clusters as the increasing thermal oxidation time. This could be explained through the hydrogen bond interactions between the $-\mathrm{OH} /-\mathrm{COOH}$ groups on the surfaces of CQDs, in consideration of the molecular characteristics of PEG. As the thermal oxidation time increased, the growing number of - $\mathrm{COOH}$ groups facilitated the hydrogen bond interactions. From the high magnification images (Figs. 3(e)-3(h)), the mean size of carbon dots is about $2 \mathrm{~nm}$, which tends to decrease with the increase of thermal oxidation time. The smaller carbon dots in PEG-110h nanofluid may be associated with their higher carbonization degree that implies more compact carbon dots. Thus, it is demonstrated that the thermally-treated PEGs are actually PEG-CQDs nanofluids with varying chemical composition. That is to say, the thermally-treated PEGs contain a great quantity of PEG-derived CQDs.

FTIR spectra were measured to provide an insight into the chemical evolution that took place during oxidation treatment. As shown in Fig. 4(a), an additional absorption band appears at $1,720 \mathrm{~cm}^{-1}$, which is gradually enhanced with the increase of thermal treatment time, corresponding to the stretching vibration of $\mathrm{C}=\mathrm{O}$ groups. Considering the enhanced $-\mathrm{OH}$ absorption band at $3,380 \mathrm{~cm}^{-1}$, it is thought that the $-\mathrm{COOH}$ groups may mainly contribute to the $1,720 \mathrm{~cm}^{-1}$ absorption band. The band at $1,643 \mathrm{~cm}^{-1}$ is usually due to the stretching mode of $\mathrm{C}=\mathrm{C}$ and the bending mode of $\mathrm{O}-\mathrm{H}$ bonds in water molecules. Since there is no characteristic absorption of the $\mathrm{C}-\mathrm{H}$ bond of double bond carbon between 3,050 and $3,100 \mathrm{~cm}^{-1}$, the absorption at $1,643 \mathrm{~cm}^{-1}$ herein should be originated from the $\mathrm{O}-\mathrm{H}$ bonds in water molecules due to the high hydrophilic performance of PEG. Note that the band at $1,643 \mathrm{~cm}^{-1}$ is more prominent

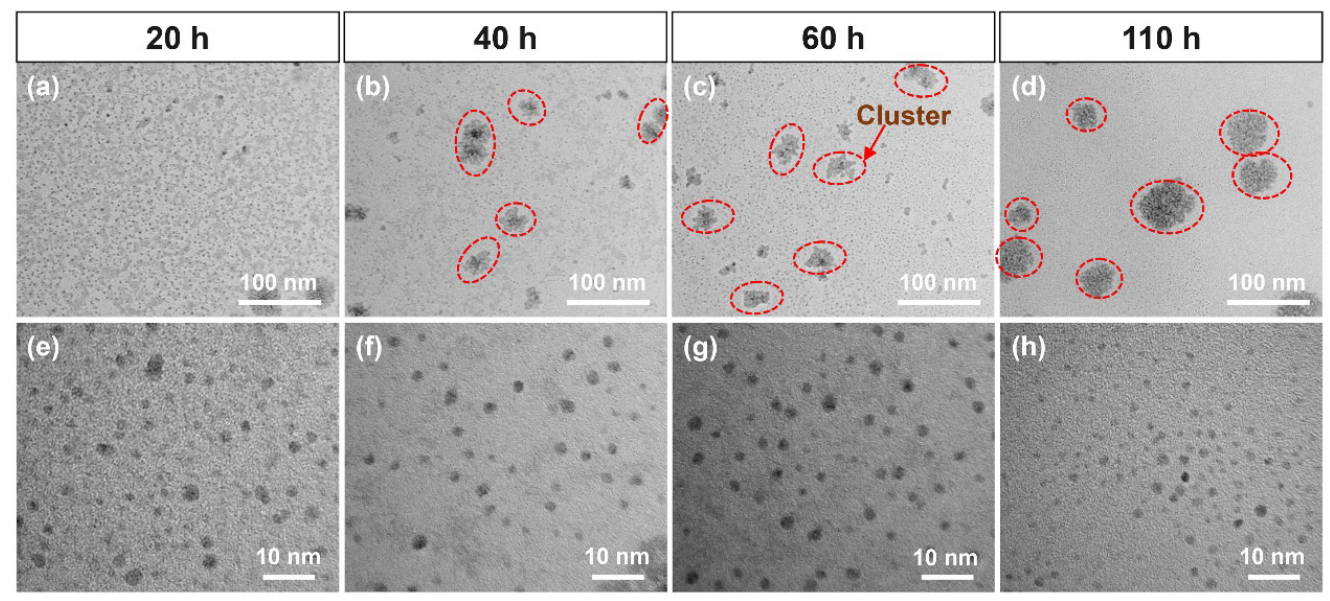

Fig. 3 TEM images of the treated PEG samples: (a) and (e) PEG-20h, (b) and (f) PEG-40h, (c) and (g) PEG-60h, (d) and (h) PEG-110h. Red dashed marks in (b), (c), and (d) refer to the CQDs clusters. 

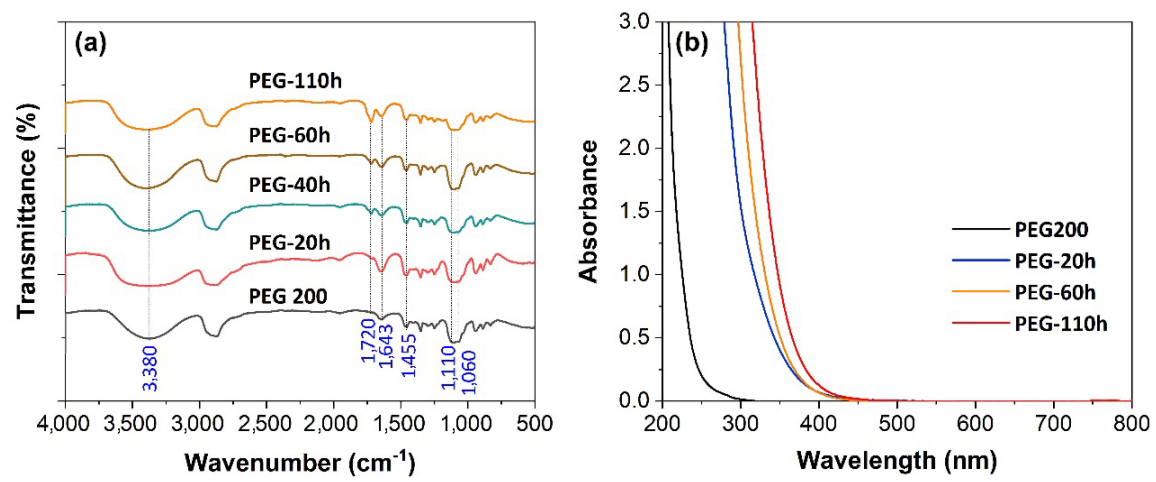

Fig. 4 (a) FTIR and (b) ultraviolet-visible absorbance spectra of PEG200 and treated PEGs.

for PEG-CQDs nanofluids, implying the increased hydrophilicity for PEG-CQDs nanofluids. Moreover, ultraviolet-visible absorbance spectra in Fig. 4(b) show that the PEG nanofluids had a significantly enhanced absorbance between 250 and $350 \mathrm{~nm}$. This demonstrated the existence of $n-\pi^{*}$ and $\pi-\pi^{*}$ transitions. Thus, it is clear that the thermally-treated PEG nanofluids contain the PEG-derived CQDs and oxidized PEG molecules. Moreover, considering the molecular characteristics of PEG, these PEG-derived CQDs should carry a lot of $-\mathrm{OH}$ groups and some $-\mathrm{COOH}$ groups on their surfaces.
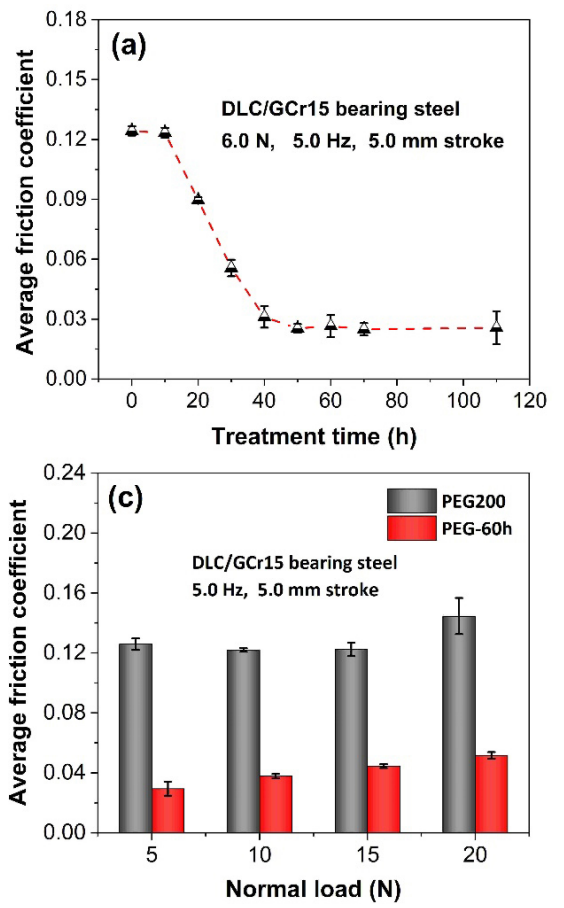

The lubricating property of the PEG200 and PEGCQDs nanofluids was evaluated through a reciprocating sliding mode at DLC film/bearing steel interfaces. As shown in Fig. 5(a), the average friction coefficient of DLC/bearing steel contact under PEG200 lubrication is around 0.12, which significantly decreases as the thermal oxidation time increases. With thermal oxidation more than $40 \mathrm{~h}$, the average friction coefficient of the system decreases by around $80.6 \%$, achieving an ultra-low sliding friction $(\mu<0.03)$. A slightly increased friction stability is noted for PEG-110h, possibly associated with its higher oxidation degree that
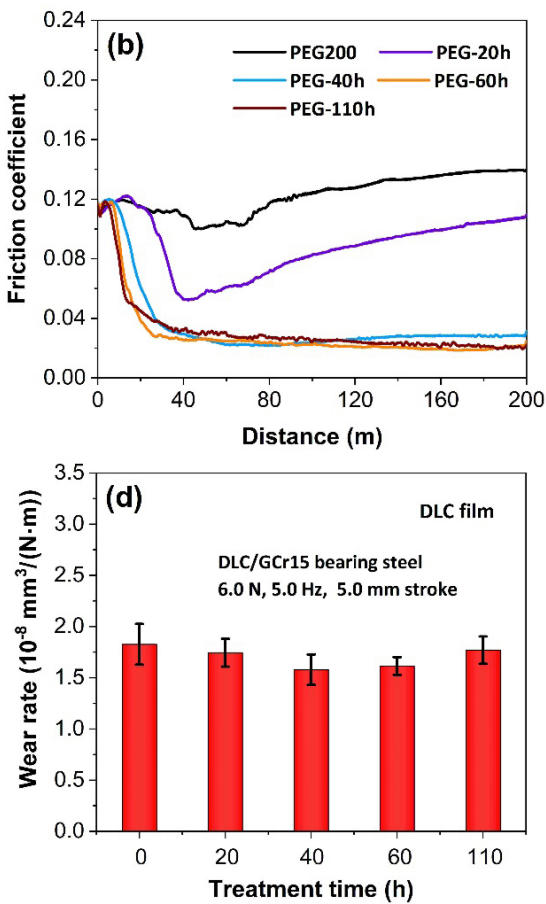

Fig. 5 (a) Average friction coefficient and (b) friction curves under original and treated PEG lubrications. (c) Average friction coefficient under PEG200 and PEG-60h lubrications at different loads. (d) Wear rates of DLC film under original and treated PEG lubrications at a load of $6 \mathrm{~N}$. 
complicates the tribochemical processes. Figure 5(b) shows the friction curves of DLC/bearing steel contacts lubricated with PEG200 and PEG-CQDs nanofluids in one of the three independent experiments. After about $30 \mathrm{~m}$ running in, PEG-CQDs nanofluids with thermal oxidation exceeding $40 \mathrm{~h}$ exhibit the ultra-low steady-state friction coefficients, around 0.02. This indicates that the slow thermal oxidation treatment is a very effective way to improve the lubricity of PEG200 at DLC/bearing steel interface. The generated PEG-CQDs nanofluid is an excellent lubricant for DLC/bearing steel contacts.

To further evaluate the properties of PEG-CQDs nanofluids, Fig. 5(c) compares the average friction coefficient of PEG200 and PEG-60h nanofluid under varying loads $(5,10,15$, and $20 \mathrm{~N}$, corresponding to maximum Herzian contact pressure 1.02, 1.28, 1.46, and 1.61 GPa, respectively). Although a mild increase is observed as the increase of contact pressure, the PEG-60h nanofluids still exhibit excellent lubricity within the applied load range, much better than PEG200. The excellent wear resistance is a key characteristic of DLC films. In solid-liquid synergy lubrication, DLC films supply not only the low friction under boundary conditions, but also the enhanced surface wear resistance, which is vital to the durability of components. It is not expected that the wear rate of DLC film increases as the thermal oxidation of PEG200. Figure 5(d) shows the specific wear rates of DLC film against GCr15 steel balls under PEG200 and nanofluid lubrications. It is evident that DLC film exhibits much lower wear rates (less than $2 \times 10^{-8} \mathrm{~mm}^{3} /(\mathrm{N} \cdot \mathrm{m})$ ) under PEG fluid lubrications than the values (ca. $\left.10^{-7} \mathrm{~mm}^{3} /(\mathrm{N} \cdot \mathrm{m})\right)$ in dry sliding contacts [31, 33]. More importantly, the nanofluids inherit the anti-wear characteristics of PEG200 at DLC film/bearing steel interface. Namely, the thermal oxidation treatment has little influence on the anti-wear property of PEG200.

After friction tests, the worn surfaces of both DLC films and steel balls were observed to infer the possible friction-reducing mechanism of PEG-CQDs nanofluids at DLC film/bearing steel interface. Figure 6 shows the wear scars on the steel balls, the sliding tracks, and their cross-section profiles on DLC surfaces. The sliding tracks formed on DLC films became wider when the PEG-CQDs nanofluids were used as lubricants. Similar size change was also seen on steel ball surfaces. Noted that the wide tracks on DLC surface lubricated with PEG-CQDs nanofluids had similar specific wear rates to that lubricated with original PEG200 (Fig. 5(d)). Thus, the widened wear tracks should correspond to the increased wear of steel balls. This means that the PEG-CQDs nanofluid is a suitable lubricant for DLC/bearing steel contact, although it mildly increases the wear of steel ball. Considering the thermal oxidation treatment, the increased steel ball wear could be associated with the oxidative species $(-\mathrm{COOH})$ in nanofluids, which caused corrosion wear events. For PEG-110h nanofluid with the highest content of oxidative species, the obvious quantity of corrosion wear products was firmly adhered around the wear scar on the surface of steel ball, as shown in Fig. 6(d). The obvious abrasion grooves were observed on both scar and track under PEG200 lubrication (Figs. 6(a) and 6(e)), but they seemingly became smoother under PEG-CQDs nanofluid lubrications (Figs. 6(i)-6(1)). This suggested that the PEG-CQDs nanofluids could effectively inhibit the abrasive friction at DLC film/bearing steel interface, and hence contribute to the ultra-low friction. As a result of increased steel ball wear, it should also be noted that the increased contact area with PEG-CQDs nanofluid lubrication reduces the contact pressure per unit area. This promotes the fluid film lubrication regime of PEG liquids to some extent.

Generally, the low friction coefficient and excellent wear resistance of DLC films are associated with the formation of a graphite-like carbon film on counterpart surface and the passivation of $\mathrm{C}-\sigma$ bonds on DLC surfaces [34, 35]. The graphitized DLC surface and the graphite-like transfer film on the counterface are frequently observed in dry contacts. However, the presence of lubricant usually inhibits the formation of a graphite-like transfer film on the contact counterface under lubricated conditions. Here, we examined the contact surfaces by Raman spectroscopy. As shown in Fig. 7(a), all Raman spectra taken from DLC surfaces show a broad, asymmetric peak between 900 and $1,800 \mathrm{~cm}^{-1}$, which is generally decomposed into D peak and $G$ peak [36]. The D peak is due to the bond stretching of $\mathrm{sp}^{2}$ carbon in rings and the $\mathrm{G}$ peak is due to the bond stretching of $\mathrm{sp}^{2}$ carbon in both rings and chains. Compared to the unworn DLC film, 

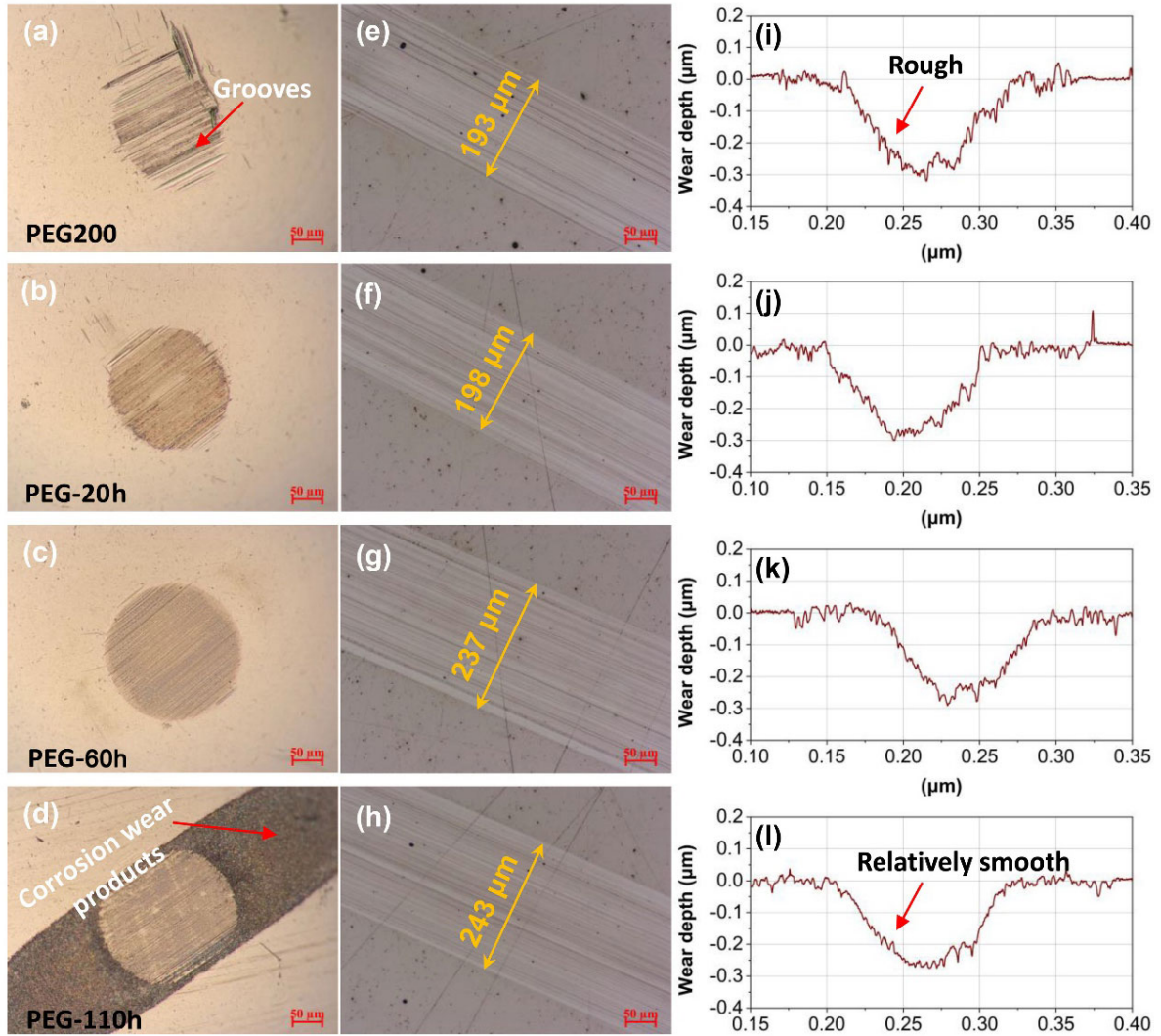

(g)
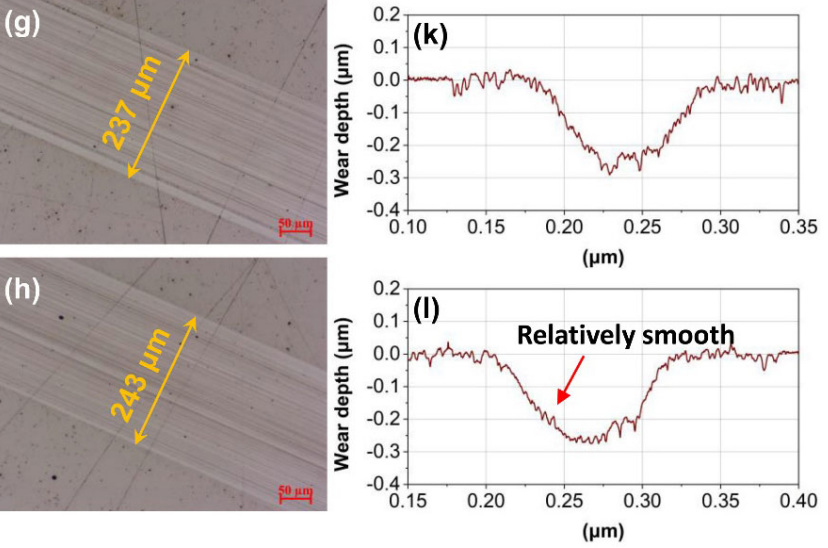

Fig. 6 Photographs of (a-d) wear scars formed on steel ball surfaces and $(\mathrm{e}-\mathrm{h})$ wear tracks formed on DLC surface under original and treated PEG lubrications. (i-l) Cross-section profiles of wear tracks (e-h) on DLC film.
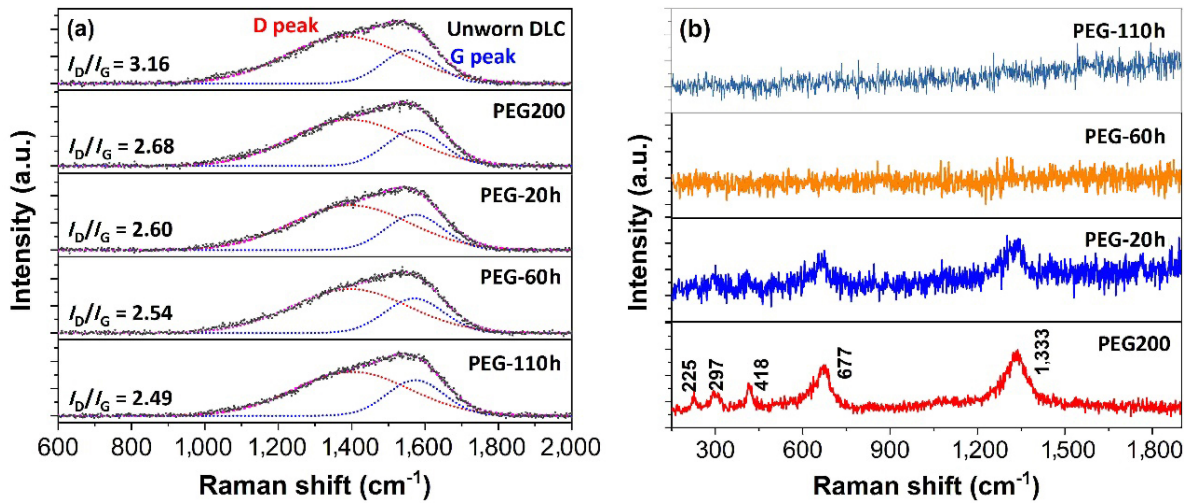

Fig. 7 Raman spectra taken from (a) worn surfaces of DLC films and (b) scars formed on steel balls under original and treated PEG lubrications.

the $I_{\mathrm{D}} / I_{\mathrm{G}}$ values from worn DLC surfaces gradually decreased with the thermal oxidation time. This often hints a decrease of $\mathrm{sp}^{2}$ hybrid carbon, suggesting that surface passivation mechanism should play an increasing role under PEG-CQDs nanofluid lubrications. It can be explained by the tribochemical reactions between DLC surface and nanofluid lubricants because the CQDs and oxidized PEG species in nanofluids contain more $-\mathrm{OH}$ and $-\mathrm{COOH}$ groups that facilitate the termination of dangling $\mathrm{C}-\sigma$ bonds on DLC surface layer. In contrast, no carbon Raman signal was observed on the worn steel ball surfaces, as shown in Fig. 7(b). But iron oxides were generated on steel ball surfaces under PEG200 lubrication, which significantly reduced under PEG-20h lubrication and vanished under PEG-60h and PEG-110h lubrications. According 
to the Raman spectra, these iron oxides are mainly composed of magnetite and hematite [37]. So, it can be concluded that the PGE-CQDs nanofluids could effectively inhibit the tribo-oxidation of steel counterface, which can cause the abrasive friction.

In addition to the above concerns, a further discussion is necessary to understand the ultra-low friction behavior of PEG-CQDs nanofluids at DLC/ bearing steel interface. In previous study, Martin et al. demonstrated that the friction-induced hydroxylation of carbon surface is the major origin of ultra-low friction of DLC film under glycerol lubrication [38]. Herein, both PEG200 and PEG-CQDs nanofluids are hydroxyl-rich lubricants and the PEG-CQDs nanofluids contain a lot of poly-hydroxyl carbon dots and oxidized PEG species (-COOH). Thus, it can be concluded that the tribochemically induced surface hydroxylation of DLC film is also crucial for the boundary lubrication of PEG200 and nanofluids. For PEG200, the -OH groups on PEG chains are insufficient to induce a highly hydroxylated contact surface, and only a partially hydroxylated interface is produced. Therefore, PEG molecules could not build an effective adsorption layer on both surfaces. A direct contact between DLC film and bearing steel causes a relatively high friction (Fig. 5(a)) and the tribo-oxidation takes place on the steel ball surface (Fig. 7(b)). After the thermal oxidation, poly-hydroxyl carbon dots and oxidized PEG species (-COOH) are generated, which are more inclined to deposit and/or be bonded to DLC surface via the tribochemical reactions. This can change the inert DLC surface into a -OH-rich carbon surface and effectively promote the adsorption of
PEG molecules on DLC surface through hydrogen bond interactions, hence building an effective PEG lubricating film on DLC surface. Due to higher reactivity, the $-\mathrm{COOH}$ groups in nanofluids may be essential to the chemical modification of friction surfaces. A transition from the high friction of PEG-20h to the low friction of PEG-40h likely confirms this because a clear $-\mathrm{COOH}$ peak is observed in the FTIR spectrum of PEG-40h, not in that of PEG-20h (Fig. 4(a)). Furthermore, the poly-hydroxyl CQDs and oxidized PEG species in nanofluids have higher tribochemical activity and stronger adsorption capacity to steel surface in contrast to original PEG200, similarly promoting the establishment of a lubricous PEG film. This may also explain the tribo-oxidation inhibition of steel surface in nanofluids. In brief, the CQDs and - $\mathrm{COOH}$ species in PEG nanofluids induce a highly hydroxylated interface in DLC/bearing steel contact via the tribochemical reactions between them and friction surfaces. Together with the mild corrosion wear and tribo-oxidation inhibition, the hydroxylated interface facilitates an effective PEG liquid film, which tends to shift a boundary lubrication to a mixed/film lubrication regime and thereby significantly reduces the friction coefficient (Fig. 8).

A further experiment was carried out to test the PEG-CQDs nanofluids at other contact interfaces, including DLC/Brass, DLC/316L stainless steel, $\mathrm{DLC} / \mathrm{ZrO}_{2}$ ceramic, and GCr15/GCr15. As shown in Fig. 9, PEG-CQDs nanofluids did not perform well in these interfaces, as that in DLC/GCr15 interface. It was even found that PEG200 exhibited better lubricity than PEG-60h in DLC/ZrO $\mathrm{rO}_{2}$ contact. Based

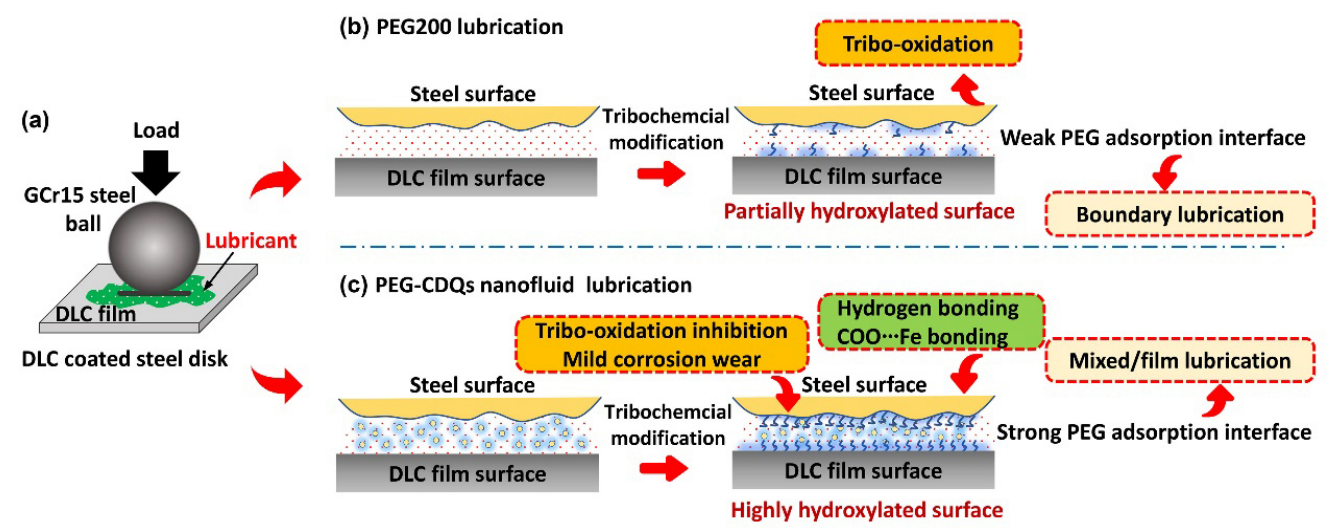

Fig. 8 Proposed friction-reducing mechanism for PEG200 and PEG-CDQs nanofluids: (a) friction model, (b) PEG200 lubrication, and (c) PEG-CQDs nanofluid lubrication. 


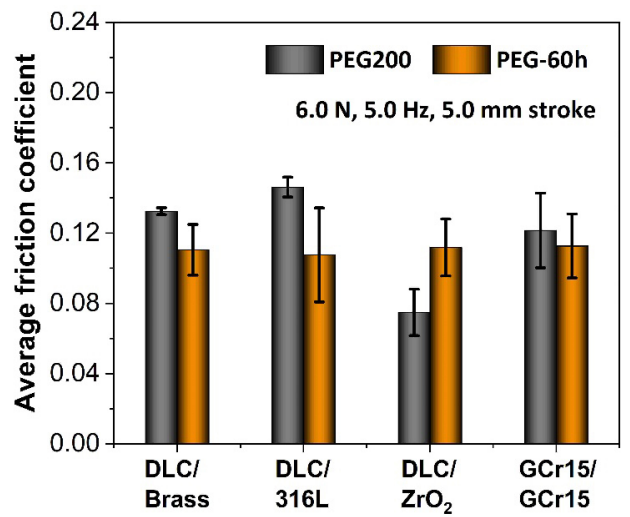

Fig. 9 Friction coefficients of DLC/Brass, DLC/316L stainless steel, DLC/ZrO 2 , and GCr15/GCr15 contacts under PEG200 and PEG-60h nanofluids lubrication.

on the friction-reducing mechanism, it is easy to understand the performance change in different contacts. For inert brass, stainless steel (chromium oxide layer), and $\mathrm{ZrO}_{2}$ surfaces, the tribochemical reactions between them and $\mathrm{CQDs},-\mathrm{COOH}$ species were inhibited. The partially hydroxylated surfaces prevented the formation of an effective PEG adsorption layer and thereby the friction-reducing mechanism failed. The high friction of GCr15/GCr15 contact indicated that DLC surface is crucial for the ultra-low friction of PEG-CQDs nanofluids because it allows a highly hydroxylated contact surface. As a result, these experiments further support the proposed friction-reducing mechanism of PEG nanofluids at DLC/GCr15 interface.

\section{Conclusions}

In summary, PEG-derived carbon quantum dots nanofluids were synthesized via a slow thermal oxidation process. The nanofluids show strong blue fluorescence emission and contain a large quantity of poly-hydroxyl C-dots and oxidized PEG species. When used as lubricant in a DLC/bearing steel interface, PEG-CQDs nanofluids showed quite excellent lubricity, achieving an ultra-low friction coefficient of around 0.02. It is concluded that the poly-hydroxyl C-dots and oxidized species $(-\mathrm{COOH})$ in nanofluids can induce a hydroxyl-rich sliding interface via the tribochemical reactions between them and friction surfaces, which promotes the adsorption of PEG molecules on the sliding surfaces. Along with the mild corrosion wear and tribo-oxidation inhibition of steel counterface, this facilitates a more effective PEG lubricating film on the contact surfaces, hence shifting the boundary lubrication to a mixed/film lubrication regime and achieving the excellent lubricity. The PEG-derived nanofluid means an excellent candidate lubricant for solid-liquid synergy lubrication based on DLC films.

\section{Acknowledgements}

This work was financially supported by the National Natural Science Foundation of China (No. 51805519), and the Natural Science Foundation for Distinguished Young Scholars of Gansu Province (No. 20JR5RA572).

Open Access This article is licensed under a Creative Commons Attribution 4.0 International License, which permits use, sharing, adaptation, distribution and reproduction in any medium or format, as long as you give appropriate credit to the original author(s) and the source, provide a link to the Creative Commons licence, and indicate if changes were made.

The images or other third party material in this article are included in the article's Creative Commons licence, unless indicated otherwise in a credit line to the material. If material is not included in the article's Creative Commons licence and your intended use is not permitted by statutory regulation or exceeds the permitted use, you will need to obtain permission directly from the copyright holder.

To view a copy of this licence, visit http://creativecommons.org/licenses/by/4.0/.

\section{References}

[1] Holmberg K, Erdemir A. Influence of tribology on global energy consumption, costs and emissions. Friction 5(3): 263-284 (2017)

[2] Meng Y, Xu J, Jin Z, Prakash B, Hu Y. A review of recent advances in tribology. Friction 8(2): 221-300 (2020)

[3] Zhang L, Xie G, Wu S, Peng S, Zhang X, Guo D, Wen S, Luo J. Ultralow friction polymer composites incorporated with monodispersed oil microcapsules. Friction 9(1): 29-40 (2019)

[4] Zhu L, Dong J, Zeng Q. High temperature solid/liquid lubrication behaviours of DLC films. Lubr Sci 33(5): 229-245 (2021) 
[5] Fan X Q, Xue Q J, Wang L P. Carbon-based solid-liquid lubricating coatings for space applications-A review. Friction 3(3): 191-207 (2015)

[6] Paskvale S, Remškar M, Čekada M. Tribological performance of TiN, TiAlN and CrN hard coatings lubricated by $\mathrm{MoS}_{2}$ nanotubes in Polyalphaolefin oil. Wear 352-353: 72-78 (2016)

[7] Kim D-W, Kim K-W. Tribological characteristics of $\mathrm{Cr} / \mathrm{CrN} / \mathrm{a}-\mathrm{C}: \mathrm{H}: \mathrm{W} / \mathrm{a}-\mathrm{C}: \mathrm{H}$ coating under boundary lubrication conditions with glycerol mono-oleate (GMO) and molybdenum dithiocarbamate (MoDTC). Wear 342-343: 107-116 (2015)

[8] Lorenzo-Martin C, Ajayi O, Erdemir A, Wei R. Tribological performance of quaternary $\mathrm{CrSiCN}$ coatings under dry and lubricated conditions. Wear 376-377: 1682-1690 (2017)

[9] Robertson J. Diamond-like amorphous carbon. Mater Sci Eng R 37(4-6): 129-281 (2002)

[10] Kano M, Yasuda Y, Okamoto Y, Mabuchi Y, Hamada T, Ueno T, Ye J, Konishi S, Takeshima S, Martin J M, De Barros Bouchet M I, Mognee T L. Ultralow friction of DLC in presence of glycerol mono-oleate (GNO). Tribol Lett 18(2): 245-251 (2005)

[11] Kalin M, Velkavrh I, Vižintin J, Ožbolt L, Review of boundary lubrication mechanisms of DLC coatings used in mechanical applications. Meccanica 43(6): 623-637 (2008)

[12] Kano M, Yoshida K. Ultra low friction of DLC coating with lubricant. J Phys: Conf Ser 258: 012009 (2010)

[13] Vengudusamy B, Mufti R A, Lamb G D, Green J H, Spikes $\mathrm{H}$ A. Friction properties of DLC/DLC contacts in base oil. Tribol Int 44(7-8): 922-932 (2011)

[14] Makowski S, Weihnacht V, Schaller F, Leson A. Ultra-low friction of biodiesel lubricated ta-C coatings. Tribol Int 71: 120-124 (2014)

[15] Yan M, Wang X, Zhang S, Zhang S, Sui X, Li W, Hao J, Liu W, Friction and wear properties of GLC and DLC coatings under ionic liquid lubrication. Tribol Int 143: 106067 (2020)

[16] Kano M, Martin J M, Yoshida K, De Barros Bouchet M I. Super-low friction of ta-C coating in presence of oleic acid. Friction 2(2): 156-163 (2014)

[17] Komori K, Umehara N. Effect of surface morphology of diamond-like carbon coating on friction, wear behavior and tribo-chemical reactions under engine-oil lubricated condition.

Tribol Int 84: 100-109 (2015)

[18] Björling M, Shi Y. DLC and Glycerol: Superlubricity in rolling/sliding elastohydrodynamic lubrication. Tribol Lett 67(1): (2019)

[19] Tasdemir H A, Tokoroyama T, Kousaka H, Umehara N, Mabuchi Y. Friction and wear performance of boundarylubricated DLC/DLC contacts in synthetic base oil. Procedia Eng 68: 518-524 (2013)
[20] Topolovec-Miklozic K, Lockwood F, Spikes H. Behaviour of boundary lubricating additives on DLC coatings. Wear 265(11-12): 1893-1901 (2008)

[21] De Barros Bouchet M I, Kano M. Superlubricity of diamond/glycerol technology applied to automotive gasoline engines. In Superlubricity. 2007: 471-492.

[22] Wang B, Tang W, Lu H, Huang Z. Ionic liquid capped carbon dots as a high-performance friction-reducing and antiwear additive for poly(ethylene glycol). J Mater Chem A 4(19): 7257-7265 (2016)

[23] Greaves M R. Polyalkylene Glycols. In Synthetics, Mineral Oils, and Bio-Based Lubricants. Rudnick L R. 3td. Boca Raton: CRC Press. 2020: 119-146.

[24] Mou Z, Wang B, Lu H, Dai S, Huang Z. Synthesis of poly(ionic liquid)s brush-grafted carbon dots for highperformance lubricant additives of polyethylene glycol. Carbon 154: 301-312 (2019)

[25] Mou Z, Wang B, Lu H, Quan H, Huang Z. Branched polyelectrolyte grafted carbon dots as the high-performance friction-reducing and antiwear additives of polyethylene glycol. Carbon 149: 594-603 (2019)

[26] Ghaednia H, Hossain M S, Jackson R L. Tribological performance of silver nanoparticle-enhanced polyethylene glycol lubricants. Tribol Trans 59(4): 585-592 (2016)

[27] Shang W, Cai T, Zhang Y, Liu D, Liu S. Facile one pot pyrolysis synthesis of carbon quantum dots and graphene oxide nanomaterials: All carbon hybrids as eco-environmental lubricants for low friction and remarkable wear-resistance. Tribol Int 118: 373-380 (2018)

[28] Tu Z, Hu E, Wang B, David K D, Seeger P, Moneke M, Stengler $\mathrm{R}, \mathrm{Hu} \mathrm{K}, \mathrm{Hu} \mathrm{X}$. Tribological behaviors of Ni-modified citric acid carbon quantum dot particles as a green additive in polyethylene glycol. Friction 8(1): 182-197 (2019)

[29] Fan R J, Sun Q, Zhang L, Zhang Y, Lu A H. Photoluminescent carbon dots directly derived from polyethylene glycol and their application for cellular imaging. Carbon 71: 87-93 (2014)

[30] Peng Z L, Ji C Y, Zhou Y Q, Zhao T S, Leblanc R M. Polyethylene glycol (PEG) derived carbon dots: Preparation and applications. Appl Mater Today 20: 100677 (2020)

[31] Xu P, Cao X, Zhang M, Yue W, Zhang G. Friction and wear behaviors of different DLC films sliding against $\mathrm{SiC}$ and $\mathrm{Si}_{3} \mathrm{~N}_{4}$ balls under high relative humidity. Diamond Relat Mater 108: 107977 (2020)

[32] Chen M, Wang W, Wu X. One-pot green synthesis of watersoluble carbon nanodots with multicolor photoluminescence from polyethylene glycol. J Mater Chem B 2(25): $3937-$ 3945 (2014) 
[33] He D, He C, Li W, Shang L, Wang L, Zhang G. Tribological behaviors of in-situ textured DLC films under dry and lubricated conditions. Appl Surf Sci 525: 146581 (2020)

[34] Liu Y, Erdemir A, Meletis E I. A study of the wear mechanism of diamond-like carbon films. Surf Coat Technol 82(1-2): 48-56 (1996)

[35] Donnet C, Fontaine J, Grill A, Le Mogne T. The role of hydrogen on the friction mechanism of diamond-like carbon films. Tribol Lett 9(3/4): 137-142 (2001)

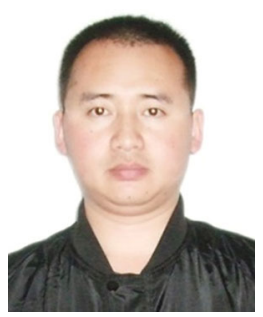

Fu WANG. He received his Ph.D. degree from Lanzhou Institute of Chemical Physics, Chinese Academy of Sciences in 2017. Then, he has been working in the State Key

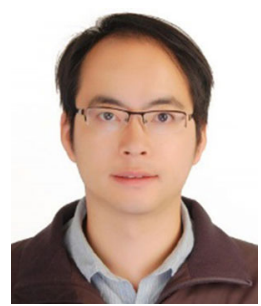

Zhaofeng WANG. He received his B.S. and Ph.D. degrees from Lanzhou University in 2006 and 2011, respectively. After his postdoctoral training at Texas State University and University of Connecticut, he
[36] Ferrari A C, Determination of bonding in diamond-like carbon by Raman spectroscopy. Diamond Relat Mater 11(3-6): 1053-1061 (2002)

[37] de Faria D L A, Venâncio Silva S, de Oliveira M T. Raman microspectroscopy of some iron oxides and oxyhydroxides. J Raman Spectrosc 28(11): 873-878 (1997)

[38] Martin J-M, Bouchet M-I D B, Matta C, Zhang Q, Goddard W A, Okuda S, Sagawa, T. Gas-phase lubrication of ta-C by glycerol and hydrogen peroxide. experimental and computer modeling. J Phys Chem C 114(11): 5003-5011 (2010)

Laboratory of Solid Lubrication at Lanzhou Institute of Chemical Physics. His research interests are the tribology of carbon-based materials and lubricant additives.

joined the State Key Laboratory of Solid Lubrication at Lanzhou Institute of Chemical Physics in 2016 as an associate professor, and was promoted to professor in 2019. His research interests focus on the intelligent lubricant materials. 